\title{
CONFORMAL TRANSFORMATIONS OF KAEHLER MANIFOLDS
}

\author{
BY S. I. GOLDBERG ${ }^{1}$ \\ Communicated by S. Bochner, November 18, 1959
}

1. By studying the pure conformal transformations of compact Kaehler manifolds ${ }^{2}$ Lichnerowicz [3] was able to prove that the largest connected Lie group $G$ of conformal transformations of an Einstein-Kaehler space $M^{2 k}(k>1)$ of positive Ricci curvature leaves invariant the Kaehlerian structure. ${ }^{3}$ Indeed, the Lie algebra $L$ of $G$ may be expressed as the direct sum of the subalgebra $K$ of the infinitesimal motions and of the space of the pure infinitesimal conformal transformations. Moreover, the pure forms on a compact Kaehler manifold are harmonic and, therefore, since the Ricci curvature is positive, it follows from a result of Bochner that there are no pure conformal transformations [1]. The above result holds also in case the Ricci curvature vanishes. Hence, since there are no infinitesimal conformal transformations if the curvature is negative the above statement holds for any compact Einstein-Kaehler space. We remark, however, that a complete Einstein space which admits a global 1-parameter group of conformal transformations must necessarily be a simply connected space of positive constant curvature [4]. The result of Lichnerowicz is particularly interesting in the study of homogeneous spaces, for, every homogeneous Kaehler space admits, if the group $G$ is semi-simple, an invariant Einstein-Kaehler metric.

The invariance of the Kaehler structure follows from Lemma 1. However, we prove that the harmonic forms of degree $p=n / 2$ of a compact and orientable Riemannian manifold $M^{n}$ of even dimension $n$ are invariant under the Lie algebra $L$ and it is this fact which allows us to prove that

The largest connected Lie group of conformal transformations of a $4 k$-dimensional compact Kaehler manifold coincides with the largest connected group of automorphisms.

By an automorphism of a Kaehler manifold is meant an analytic homeomorphism of the manifold on itself which is an isometry. The proof depends essentially on formula (6) below.

1 This research was supported by the United States Air Force Office of Scientific Research of the Air Research and Development Command, under Contract No. AF-49(638)-14.

2 The manifolds, differential forms and tensorfields considered are assumed to be of class $C^{\infty}$.

3 This was also obtained by Yano and Nagano. 
2. Let $\alpha$ and $\beta$ be any two $p$-forms on a compact and orientable Riemannian manifold $M^{n}$ and denote by $X$ an infinitesimal transformation on $M^{n}$. Then, by Stokes' theorem and the fact that

$$
\begin{aligned}
\theta(X) & =d i(X)+i(X) d, \\
\int_{M^{n}} \theta(X)\left(\alpha \wedge^{*} \beta\right) & =\int_{M^{n}} d i(X)\left(\alpha \wedge^{*} \beta\right)=0
\end{aligned}
$$

where $\theta(X), i(X)$ and $d$ denote the operations of Lie derivation, contraction (interior product) and exterior derivation, respectively and * denotes the star operation of Hodge. Since $\theta(X)$ is a derivation,

$$
(\theta(X) \alpha, \beta)=-\int_{M^{n}} \alpha \wedge \theta(X) * \beta .
$$

If, therefore, we put

$$
* \bar{\theta}(X) \beta=-\theta(X) * \beta,
$$

that is

$$
\bar{\theta}(X)=(-1)^{n p+p+1} * \theta(X) *,
$$

we have

$$
(\theta(X) \alpha, \beta)=(\alpha, \bar{\theta}(X) \beta) .
$$

It follows that the operator $\bar{\theta}(X)$ is the dual of $\theta(X)$. One therefore obtains

$$
\bar{\theta}(X)=\delta \epsilon(\xi)+\epsilon(\xi) \delta
$$

where $\xi=X_{i} d x^{i}$ is the covariant form for $X$ and $\epsilon(\xi)$ is the dual of $i(X)$ :

$$
i(X)=(-1)^{n(p-1)} * \epsilon(\xi) *,
$$

that is

$$
\epsilon(\xi) \alpha=\xi \wedge \alpha
$$

for any $p$-form $\alpha$. The operators $\theta(X)$ and $d$ commute and, clearly, so do their duals as one may also infer from (3). Moreover, if $g$ denotes the metric tensor of $M^{n}$

$$
\begin{aligned}
(\theta(X)+\bar{\theta}(X)) \alpha=\alpha \cdot \delta \xi+ & \sum_{r=1}^{p} g^{j k}(\theta(X) g)_{k i_{r}} \\
& \cdot \alpha_{i_{1} \cdots i_{r-1} j i_{r+1} \cdots i_{p}} d x^{i_{1}} \wedge \cdots \wedge d x^{i_{p}}
\end{aligned}
$$


LEMMA 1. The harmonic forms on a compact and orientable Riemannian manifold are $K$-invariant differential forms.

The proof depends on the fact that $\theta(X)+\bar{\theta}(X), X \in K$ annihilates harmonic forms [2]. Since $X$ is an infinitesimal motion, $\theta(X) g=0$ from which it follows that $\delta \xi$ vanishes also. If $\alpha$ is a harmonic form $d \theta(X) \alpha=\theta(X) d \alpha=0$ and $\delta \theta(X) \alpha=-\delta \bar{\theta}(X) \alpha=-\bar{\theta}(X) \delta \alpha=0$. Hence $\theta(X) \alpha$ is a harmonic form. But $\theta(X) \alpha=d i(X) \alpha$ from which by the Hodge decomposition of a form $\theta(X) \alpha=0$.

Let $X$ be an element of $L$. Then

$$
\theta(X) g=-\frac{2}{n}(\delta \xi) \cdot g,
$$

and, from (4)

$$
\theta(X) \alpha+\bar{\theta}(X) \alpha=\left(1-\frac{2 p}{n}\right)(\delta \xi) \alpha .
$$

We therefore obtain the following partial generalization of Lemma 1:

LEMma 2. The harmonic forms of degree $p=n / 2$ of a compact, orientable Riemannian manifold $M$ of even dimension $n$ are invariant under the Lie algebra of infinitesimal conformal transformations on $M$.

Lemma 3. If $\operatorname{dim} M=2$, the inner product of a harmonic vector field and a vector field defining an infinitesimal conformal transformation is a constant on $M$.

This is clearly the case if $M$ is a Riemann surface.

Lemma 4. An infinitesimal conformal transformation $X$ of a 4-dimensional compact Kaehler manifold $M$ is necessarily a motion.

Indeed, by Lemma 2, $\theta(X) \omega=0$ where $\omega$ is the fundamental 2 -form defining the Kaehler structure. Since $\omega$ is closed, $\theta(X) \omega=d i(X) \omega$ and hence $i(X) \omega$ is closed. Thus, by the Hodge decomposition theorem $i(X) \omega=d \phi+H[i(X) \omega]$ for some (real) $C^{\infty}$ function $\phi$; the operator $H$ denotes the harmonic part. Now, $i(X) \omega=C \xi$ where $C$ is the complex structure operator applied to forms. Hence, $\xi=-C d \phi+H[\xi]$ and, therefore since $\delta C d \phi=0, \delta \xi=0$ and $X$ is an infinitesimal motion.

PROOF OF THE MAIN THEOREM. The form $\omega^{k}$ being a harmonic form of degree $2 k, \theta(X) \omega^{k}=0$ by Lemma 2 . But $\theta(X) \omega^{k}=k \theta(X) \omega \wedge \omega^{k-1}$ and so, since the map $\alpha \rightarrow \alpha \wedge \omega$ where $\alpha$ is a $p$-form defines an isomorphism from the space of $p$-forms $\Lambda^{p}(M)$ into $\Lambda^{p+2}(M)$ for $p \leqq 2 k-2$, it follows that $\theta(X) \omega$ vanishes. We may again conclude that the 
infinitesimal transformation $X$ is an infinitesimal motion. It remains to be shown that $X$ is analytic for, since $M^{4 k}$ is compact, the Lie algebra of (contravariant) analytic vector fields is the Lie algebra of the group of analytic homeomorphisms of $M^{4 k}$. Let $F_{j}^{\imath}$ denote the tensorfield of type $(1,1)$ defining the complex structure on $M^{4 k}$. We define the tensorfield $t(X)$ of type $(0,2)$ :

$$
t(X)_{i j}=X_{; k}^{i} F_{j}^{k}-X_{; j}^{k} F_{k}^{i}
$$

and remark that the condition $t(X)=0$ characterizes the (contravariant) analytic vector fields. From (7)

$$
t(X)_{i j}=F_{j}^{k}(\theta(X) g)_{i k}+(\theta(X) \omega)_{i j},
$$

and so, by Lemma 1 , since $X$ is an infinitesimal motion, $t(X)$ vanishes.

THEOREM. An infinitesimal conformal transformation of a compact Kaehler manifold $M^{2 k}(k>1)$ whose associated 1-form is closed is an automorphism of $M^{2 k}$.

Indeed, from (8) if $X$ defines an infinitesimal conformal transformation,

$$
t(X)=\theta(X) \omega-\frac{1}{k}(\delta \xi) \omega .
$$

Our notation does not distinguish between the 2 -form $t(X)$ and the tensor field $t(X)$. Since $\xi$ is closed

$$
t(X)=\theta(C \xi) g
$$

that is, $t(X)$ is a symmetric tensor. But $t(X)$ is also skew-symmetric and must therefore vanish. Hence,

$$
\theta(X) \omega=\frac{1}{k}(\delta \xi) \omega .
$$

It follows that $d \delta \xi \wedge \omega=0$ and since $k>1$, we may conclude that $d \delta \xi=0$. Now,

$$
(d \delta \xi, \xi)=(\delta \xi, \delta \xi)
$$

and so $\delta \xi$ vanishes, that is $X$ is an infinitesimal motion.

An infinitesimal conformal transformation $X$ is said to be homothetic if $d \delta \xi=0$. From the proof of the above theorem we see that a homothetic transformation of a compact Kaehler manifold is an automorphism of the manifold. 
Added in proof. The main theorem is valid for almost Kaehler manifolds as well. The proof of this fact will appear in a subsequent paper.

\section{REFERENCES}

1. S. Bochner, Vector fields and Ricci curvature, Bull. Amer. Math. Soc. vol. 52 (1946) pp. 776-797.

2. S. Bochner and K. Yano, Curvature and Betti numbers, Annals of Mathematics Studies, vol. 32, p. 49.

3. A. Lichnerowicz, Some problems on transformations of Riemannian and Kaehlerian manifolds, Mimeographed notes, Princeton, 1956.

4. K. Yano and T. Nagano, Einstein spaces admitting a one-parameter group of conformal transformations, Ann. of Math. vol. 69 (1959) pp. 451-461.

HARVARD UNIVERSITY AND

WAyne State University 